\title{
THE
}

\section{A U-shaped Association Between Blood Pressure and Cognitive Impairment in Chinese Elderly}

Yue-Bin Lv

Peng-Fei Zhu

Zhaeo-Xue Yin

Virginia Byers Kraus

Diane Threapleton

See next page for additional authors

Follow this and additional works at: https://digitalcommons.uri.edu/soc_facpubs

This is a pre-publication author manuscript of the final, published article.

Creative Commons License

(c) (1)(9)

This work is licensed under a Creative Commons Attribution-Noncommercial-No Derivative Works 4.0 License.

\section{Citation/Publisher Attribution}

Lv, Y-B, Zhu, P-F, Yin, Z-X, Kraus, V. B., Threapleton, D., Chei, C-L., Brasher, M. S.,...Shi, X-M. (2017) A Ushaped Association Between Blood Pressure and Cognitive Impairment in Chinese Elderly. Journal of the American Medical Directors Association, 18(2). 193.e7-193.e13. doi: 10.1016/j.jamda.2016.11.011 Available at: http://dx.doi.org/10.1016/j.jamda.2016.11.011

This Article is brought to you for free and open access by the Sociology \& Anthropology at DigitalCommons@URI. It has been accepted for inclusion in Sociology \& Anthropology Faculty Publications by an authorized administrator of DigitalCommons@URI. For more information, please contact digitalcommons-group@uri.edu. 


\section{Authors}

Yue-Bin Lv, Peng-Fei Zhu, Zhaeo-Xue Yin, Virginia Byers Kraus, Diane Threapleton, Choy-Lye Chei, Melanie S. Brasher, Juan Zhang, Han-Zhu Qian, Chen Mao, David Bruce Matchar, Jie-Si Luo, Yi Zeng, and Xiao-Ming Shi

This article is available at DigitalCommons@URI: https://digitalcommons.uri.edu/soc_facpubs/29 


\section{UNIVERSITY OF LEEDS}

This is a repository copy of $A U$-shaped Association Between Blood Pressure and Cognitive Impairment in Chinese Elderly.

White Rose Research Online URL for this paper:

http://eprints.whiterose.ac.uk/138915/

Version: Accepted Version

\section{Article:}

Lv, Y-B, Zhu, P-F, Yin, Z-X et al. (11 more authors) (2017) A U-shaped Association Between Blood Pressure and Cognitive Impairment in Chinese Elderly. Journal of the American Medical Directors Association, 18 (2). 193.e7-193.e13. ISSN 1525-8610

https://doi.org/10.1016/j.jamda.2016.11.011

(c) 2016 Published by Elsevier Inc. on behalf of AMDA - The Society for Post-Acute and Long-Term Care Medicine. Licensed under the Creative Commons Attribution-NonCommercial-NoDerivatives 4.0 International License (http://creativecommons.org/licenses/by-nc-nd/4.0/).

\section{Reuse}

This article is distributed under the terms of the Creative Commons Attribution-NonCommercial-NoDerivs (CC BY-NC-ND) licence. This licence only allows you to download this work and share it with others as long as you credit the authors, but you can't change the article in any way or use it commercially. More information and the full terms of the licence here: https://creativecommons.org/licenses/

\section{Takedown}

If you consider content in White Rose Research Online to be in breach of UK law, please notify us by emailing eprints@whiterose.ac.uk including the URL of the record and the reason for the withdrawal request. 


\section{A U-shaped Association between Blood Pressure and Cognitive Impairment in Chinese Elderly}

Yue-Bin Lv, M.D., ${ }^{\text {a }}$ Peng-Fei Zhu, M.D., ${ }^{\mathrm{a}}$ Zhao-Xue Yin, M.D., ${ }^{\mathrm{b}}$ Virginia Byers Kraus, M.D., Ph.D., ${ }^{\mathrm{C} D i a n e}$ Threapleton, M.D., Ph.D., ${ }^{\mathrm{d}}$ Choy-Lye Chei, Ph.D., ${ }^{\mathrm{e}}$ Melanie Sereny Brasher, Ph.D., ${ }^{\mathrm{f}}$ Juan Zhang, M.D., Ph.D., ${ }^{\mathrm{b}}$ Han-Zhu Qian, M.D., Ph.D., ${ }^{\mathrm{g}}$ Chen Mao, M.D., Ph.D., ${ }^{\mathrm{d}}$ David Bruce Matchar, M.D., Ph.D., ${ }^{\mathrm{e}, \mathrm{J}}$ Jie-Si Luo, M.D., ${ }^{\mathrm{b}}$ Yi Zeng, Ph.D., ${ }^{\mathrm{i}, \mathrm{j}}$ and XiaoMing Shi, M.D., Ph.D. ${ }^{*}$

A Institute of Environmental Health and Related Products Safety, Chinese Center for Disease Control and Prevention, Beijing, China

B Division of Non-Communicable Disease Control and Community Health, Chinese Center for Disease Control and Prevention, Beijing, China

C Duke Molecular Physiology Institute and Division of Rheumatology, Department of Medicine, Duke University School of Medicine, Durham, North Carolina, USA

D Division of Epidemiology, JC School of Public Health and Primary Care, The Chinese University of Hong Kong, Hong Kong, China

E Health Services and Systems Research, Duke-NUS Graduate Medical School Singapore, Singapore

F Department of Sociology and Anthropology; Department of Human Development and Family Studies, University of Rhode Island, Kingston, Rhode Island, USA

G Vanderbilt Institute for Global Health; Division of Epidemiology, Department of Medicine, Vanderbilt University, Nashville, Tennessee, USA

H Division of General Internal Medicine, Department of Medicine, Duke University School of Medicine, Durham, North Carolina, USA

I Center for the Study of Aging and Human Development and the Geriatric Division of School of Medicine, Duke University, Durham, North Carolina, USA

J Center for Study of Healthy Aging and Development Studies, Peking University, Beijing, China

Correspondence to: Xiao-Ming SHI, MD, PhD, Institute of Environmental Health and Related Products Safety, Chinese Center for Disease Control and Prevention, \#7 Panjiayuan Nanli, Chaoyang, Beijing 100021, China, nc.cdcanihc@mxihs, Telephone: (+86) 1050930101, Fax: (+86) 1058900247

First Author: Yue-Bin Lv, Peng-Fei Zhu and Zhao-Xue Yin, who contribute to the paper equally. 


\section{Abstract \\ Objectives}

Higher or lower blood pressure may relate to cognitive impairment, while the relationship between blood pressure and cognitive impairment among the elderly is not well-studied. The study objective was to determine whether blood pressure is associated with cognitive impairment in the elderly, and, if so, to accurately describe the association.

Design

Cross-sectional data from the sixth wave of the Chinese Longitudinal Healthy Longevity Survey (CLHLS) conducted in 2011

\section{Setting}

Community-based setting in longevity areas in China

Participants

7,144 Chinese elderly aged 65 years and older were included in the sample

\section{Measures}

Systolic blood pressures (SBP) and diastolic blood pressures (DBP) were measured, pulse pressure (PP) was calculated as (SBP)-(DBP) and mean arterial pressures (MAP) was calculated as $1 / 3(\mathrm{SBP})+2 / 3(\mathrm{DBP})$. Cognitive function was assessed via a validated MiniMental State Examination (MMSE).

\section{Results}

Based on the results of generalized additive models (GAMs), U-shaped associations were identified between cognitive impairment and SBP, DBP, PP and MAP. The cut-points at which risk for cognitive impairment (MMSE<24) was minimized were determined by quadratic models as $141 \mathrm{mmHg}, 85 \mathrm{mmHg}, 62 \mathrm{mmHg}$ and $103 \mathrm{mmHg}$, respectively. In the logistic models, U-shaped associations remained for SBP, DBP, and MAP but not PP. Below the identified cut-points, each $1 \mathrm{mmHg}$ decrease in blood pressure corresponded to $0.7 \%$, $1.1 \%$, and $1.1 \%$ greater risk in the risk of cognitive impairment, respectively. Above the cutpoints, each $1 \mathrm{mmHg}$ increase in blood pressure corresponded to $1.2 \%, 1.8 \%$, and $2.1 \%$ greater risk of cognitive impairment for SBP, DBP and MAP, respectively.

Conclusion

A U-shaped association between blood pressure and cognitive function in an elderly Chinese population was found. Recognition of these instances is important identifying the high-risk population for cognitive impairment and to individualize blood pressure management for cognitive impairment prevention.

Keywords: blood pressure, hypertension, hypotension, cognitive impairment, curvilinear association, elderly 


\section{Introduction}

Hypertension and hypotension are important risk factors for vascular diseases, which may lead to cognitive impairment due to stroke or chronic cerebral hypoperfusion in the elderly [1]. This suggests that hypertension and hypotension may both be associated with cognitive impairment, a common neurological disorder in the elderly.

In recent years, the link between blood pressure, including systolic blood pressures (SBP) and diastolic blood pressures (DBP), to cognitive function has attracted much attention, but the role of blood pressure in cognitive impairment is still unclear [2-14]. Several studies reported a linear association between elevated blood pressure and increased risk of cognitive impairment or dementia [ $\underline{2}, \underline{3}]$, and low blood pressure was related to higher risk of dementia in the elderly $[\underline{4}, 5]$. Additionally, antihypertensive medicines have a valuable role in the prevention of dementia or cognitive impairment [으, $\underline{7}]$, while some studies failed to confirm this association $[\underline{8}, \underline{9}]$.

One proposed explanation for inconsistencies among these studies is that the relationship between blood pressure and cognitive function is nonlinear. Several investigators have demonstrated a U-shaped relationship [10-14] or a J-shaped association between cognitive function and blood pressure [4]. Most related researches use linear model including multivariate regression model $[\underline{12}, \underline{15}]$, hierarchical linear model $[\underline{14}, \underline{16}]$, mixed-effects regression analyses[11] to examine the possible relations. Few studies use splines to determine presence and type of the curvilinear relationships [17]. None of the curvilinear associations were demonstrated using generalized additive models (GAMs) and quadratic models, whose strengths include the ability to determine the natural shape of the relationship, sensitivity to detect non-linear relationships and identifying the optimal cut-points between dependent and predictor variable(s).

Combined measures such as pulse pressure (PP) or mean arterial pressure (MAP) may be a salient blood pressure index in the elderly[18] as SBP continuously increases and DBP continuously decreases after age 60[19]. Notably, PP and cognitive function have not been extensively studied, with positive associations with cognitive impairment being reported in some [20-23] but not all [24] studies. Some studies suggest that MAP may be a more accurate predictor than other blood pressure indices [25, 26].

It is important to clarify whether blood pressure is associated with cognitive impairment in the elderly, and, if so, to accurately describe the association. In the present study, we investigated the relationship between blood pressure (as measured by SBP, DBP, PP and MAP) and cognitive impairment among Chinese elderly, and the possibility of a linear or curvilinear relationship using GAMs and quadratic models.

\section{Methods}

\section{Study population}

A sample of 7144 elderly aged 65 years and older was included in the sixth wave of the Chinese Longitudinal Healthy Longevity Survey (CLHLS) conducted in 2011. The details of the CLHLS and sample design have been described elsewhere[27,28]. Informed consent was obtained from all participants and/or their relatives, and the study was approved by the Ethics Committee of Peking University. 


\section{Assessment of cognitive function}

Cognitive function was assessed by the Mini-Mental State Examination (MMSE) [29], which has been widely applied in epidemiological studies. Five domains of cognitive function were included, namely orientation, registration, attention, memory, language and visual construction skills [30]. The scores ranged from 0 to 30 points, and cognitive impairment was identified if the MMSE score was less than 24 [31].

\section{Assessment of blood pressure}

After having rested for 5 minutes under supervision of trained research assistants, arterial blood pressure was measured with a mercury sphygmomanometer on the right arm at heart level of the seated subject. In our analysis, the mean value of two blood pressure measurements was calculated and used for further analyses. PP was calculated as (SBP)(DBP) and MAP was calculated as $1 / 3(\mathrm{SBP})+2 / 3(\mathrm{DBP})$. Hypertension was defined as systolic blood pressure $\geq 140 \mathrm{~mm} \mathrm{Hg}$ and/or diastolic blood pressure $\geq 90 \mathrm{~mm} \mathrm{Hg}$.

\section{Assessment of covariates}

Covariates were collected by a structured questionnaire, one part is sociodemographic characteristics including age, sex, educational background and residence; the other part is health characteristics including physical activity, measured waist circumference, and selfreported diabetes mellitus, cardiovascular diseases and stroke and other cerebrovascular diseases. Age and education were two of the most important confounders in the analysis of association between cognitive impairment and blood pressure, and were modeled as continuous variables in years. Residence was categorized as urban or rural. Physical activity was classified into "yes" or "no" by the question "Do you often participate in physical activities, including walking, playing ball, running, and Qigong?". Non-stretchable tape was used to measure waist circumference at a level between the lowest rib and iliac crest. Central obesity was identified if the participant's waist circumference was $\geq 85 \mathrm{~cm}$ in men or $\geq 80 \mathrm{~cm}$ in women.

\section{Statistical analyses}

Generalized additive models were performed to explore the linear or curvilinear association between SBP, DBP, PP, MAP and odds of having cognitive impairment. GAMs establish a relationship between the mean of a dependent variable and a smoothed (non-parametric) function of the predictor variable(s) via a link function. As GAM analysis suggested Ushaped associations between SBP, DBP, PP, MAP and risk of cognitive impairment on the basis of smoothed curve (Figure 1), quadratic regression was performed predicting risk of cognitive impairment: $\operatorname{Pr}($ Cognitive impairment $\mid$ Blood pressure $)=\left\{1+\exp \left[-\left(\alpha+\beta_{1}\right.\right.\right.$ (Blood pressure $)+\beta_{2}$ (Blood pressure $\left.\left.)^{2}\right]\right\}^{-1}$, with models parameters $\alpha, \beta_{1}$ and $\beta_{2}$ estimated using maximum likelihood methods. If $\beta_{1}$ and $\beta_{2}$ are both non-zero and statistically significant, then the estimated level of blood pressure at which risk of cognitive impairment is minimized is $\left(\beta_{1} / 2 \beta_{2}\right)$. These values are used as cut-points of blood pressure for further analysis. For SBP, DBP, PP and MAP, the $\beta_{1}$ were estimated as $-0.07908,-0.10114,-0.03585$ and -0.13279 respectively, and the $\beta_{2}$ were estimated as $0.00028,0.00059,0.00029$ and 0.00064 respectively; all $\mathrm{p}$ values were less than 0.01 . The blood pressure values associated with lowest risk were identified as $141 \mathrm{~mm} \mathrm{Hg}, 85 \mathrm{~mm} \mathrm{Hg}, 62 \mathrm{~mm} \mathrm{Hg}$ and $103 \mathrm{~mm} \mathrm{Hg}$ for SBP, DBP, PP and MAP, respectively. For each blood pressure measure, subjects were divided into 4 groups, first based on whether they were above or below the cut-point for that measure, and within these 2 groups whether they were above or below the mean value of that measure. Thus, subjects were first stratified into two groups by cut-points of each blood pressure index 
(values noted above); subjects in the low group were then divided into two sub-groups (denoted low and medium-low) using medians of each index $(127 \mathrm{~mm} \mathrm{Hg}, 77 \mathrm{~mm} \mathrm{Hg}, 49 \mathrm{~mm}$ $\mathrm{Hg}, 93 \mathrm{~mm} \mathrm{Hg}$ for SBP, DBP, PP and MAP, respectively) and subjects in the high group were divided into two sub-groups (denoted high and medium-high) using the medians of each index (155mm Hg, $90 \mathrm{~mm} \mathrm{Hg}, 73 \mathrm{~mm} \mathrm{Hg}, 110 \mathrm{~mm} \mathrm{Hg}$ for SBP, DBP, PP and MAP respectively).

Then logistic regression models were performed to assess the risk of cognitive impairment with SBP, DBP, PP and MAP as continuous or binary predictor variables below and above the cut-points. In a basic model, we adjusted for demographic variables including age, gender, years of education, and residence, and further adjusted for physical activity, central obesity, diabetes mellitus, cardiovascular diseases, stroke and cerebrovascular diseases in the final model. Odds ratios (ORs) with $95 \%$ confidence intervals (CIs) in logistic regression models were presented. SAS 9.2 (SAS Institute Inc., Cary, NC) was used for all analyses.

\section{Description of population}

The age of 7,144 participants ranged from 65 to 115 years, with an average of 87 years; $55 \%$ of participants were female. The mean SBP, DBP, PP and MAP were 135.5, 80.1, 55.4 and $98.6 \mathrm{~mm} \mathrm{Hg}$, respectively; $42.3 \%$ of participants had hypertension (Table 1). The average MMSE score was 21.1, and prevalence of cognitive impairment was 38.5\% (27.8\% for men and $47.3 \%$ for women). The participants who were men, living in urban areas, participating in physical activity, and with cardiovascular diseases were less likely to be cognitively impaired. The participants with type 2 diabetes or stroke and cerebrovascular diseases were more likely to be cognitively impaired (Table 1). The prevalence of hypertension or blood pressure measures was not significantly different in those with and without cognitive impairment.

\section{Associations between blood pressure and cognitive impairment}

The results of GAMs and quadratic models suggested a U-shaped association between SBP, DBP, PP, and MAP and cognitive impairment. Logistic models were consistent with the Ushaped associations between cognitive impairment and SBP, DBP, and MAP; however, such an association was not apparent for PP, regardless of considering PP as a continuous or categorical variable.

\section{SBP and cognitive impairment}

Among the participants whose SBP levels were below the cut-point (141 mm Hg), there was a trend towards significantly decreasing risk of cognitive impairment with increasing levels of SBP; each $1 \mathrm{~mm} \mathrm{Hg}$ lower SBP corresponded to a $1.4 \%$ higher risk of cognitive impairment before adjustment (OR, 1.014; 95\% CI, 1.009-1.019). The risk attenuation remained significant after adjusting for covariates in the basic and final models, which decreased to $0.8 \%$ (OR, 1.008; 95\% CI, 1.003-1.014) and 0.7\% (OR, 1.007; 95\% CI, $1.002-$ 1.013) respectively (Table 2). Participants whose SBP levels were below the cut-point were stratified into two equal-sized groups, the univariate analysis showed that the low SBP group had higher risk of cognitive impairment compared to medium-low SBP group (OR, 1.25; 95\% CI, 1.12-1.41). The multivariate OR (95\% CI) reported in the basic model was 1.15 (1.00-1.32) and in the final model was $1.18(1.02-1.35)$ (Table 3).

In contrast with findings below the cut-points, the risk of cognitive impairment was greater with each higher unit of SBP among the participants whose SBP levels were above the cutpoints. Each $1 \mathrm{~mm} \mathrm{Hg}$ higher SBP corresponded to a $1.2 \%$ greater risk of cognitive 
impairment in the univariate and multivariate models (Table 2). Compared with medium-high SBP group, participants with high SBP level had a 23\% higher risk of cognitive impairment in the final model (OR, 1.23; 95\% CI, 1.02-1.49) (Table 3).

\section{DBP and cognitive impairment}

Similar to the relationship seen for SBP, for DBP below the cut-points of $85 \mathrm{~mm} \mathrm{Hg}$ the increased risk of cognitive impairment was also found with lower DBP, there was a $1.1 \%$ greater risk of cognitive impairment with each $1 \mathrm{~mm} \mathrm{Hg}$ lower DBP after full adjustment (OR, 1.011; 95\% CI, 1.003-1.020). Compared with medium-low DBP group, participants with low DBP levels had a 33\% higher risk of cognitive impairment before adjustment (OR, $1.33 ; 95 \%$ CI, 1.19-1.49), and the association remained after the adjustment.

Above the cut-point of $85 \mathrm{~mm} \mathrm{Hg}$, when DBP was taken as a continuous variable, there was a $1.8 \%$ greater risk of cognitive impairment with each $1 \mathrm{~mm} \mathrm{Hg}$ increment of DBP before adjustment and full adjustment (Table 2). When higher DBP was stratified into two equal groups, participants with high DBP levels had a $24 \%$ higher risk of cognitive impairment compared with medium-high DBP group in the final model (OR, 1.24; 95\% CI, 1.02-1.52).

\section{PP and cognitive impairment}

Although we found a U-shaped association between cognitive impairment and PP in the GAMs models (Figure 1), quadratic models and as a continuous variable in the univariate logistic model, it was non-significant based on multivariate logistic regression (Table 2, Table 3).

\section{MAP and cognitive impairment}

As shown in GAMs and in quadratic models, the U-shaped association was confirmed in the logistic regression model, that is, each $1 \mathrm{~mm} \mathrm{Hg}$ lower MAP corresponded to a $1.1 \%$ greater risk in cognitive impairment below the cut-points of $103 \mathrm{~mm} \mathrm{Hg}$, and each $1 \mathrm{~mm} \mathrm{Hg}$ higher MAP corresponded to a $2.1 \%$ greater risk in cognitive impairment above the cut-points of $103 \mathrm{~mm} \mathrm{Hg}$ (Table 2). Poorer cognitive performance was found in those with low MAP level compared to those with medium-low MAP level, and in those with high MAP level compared to those with medium-high MAP level (Table 3)

\section{Discussion}

\section{U-shaped association between blood pressure and cognitive impairment}

In this cross-sectional study of 7144 Chinese elderly aged 65 years and older, we observed Ushaped associations between blood pressure (SBP, DBP, and MAP) and cognitive impairment, with extremes of blood pressures associated with poorer cognitive performance after controlling for demographic, lifestyle and biological confounding factors. This association was less evident for PP as the U-shaped relationship identified in the GAMs, quadratic models and univariate logistic models, Previous studies have provided inconsistent results, indicating linear, J-shaped or no association [2- -9$]$. Notably, one proposed explanation was that linear function based on experience rather than the natural shape between blood pressure and cognitive impairment may not adequately describe the association.

Several previous reports are consistent with our finding of a U-shaped association of blood pressure with cognitive impairment or dementia [10-14]. In a cross-sectional study conducted in a biracial community, the highest cognitive function scores were found in the elderly aged 65 and older whose SBP was at the mean of $140 \mathrm{~mm} \mathrm{Hg}$, or in the persons whose DBP was at 
the mean of $77 \mathrm{~mm} \mathrm{Hg} \mathrm{[10].} \mathrm{Similarly,} \mathrm{this} \mathrm{phenomenon} \mathrm{was} \mathrm{also} \mathrm{confirmed} \mathrm{in} \mathrm{a} \mathrm{9-year}$ longitudinal study in which a U-shaped association with higher risk of cognitive impairment among individuals with higher $(\geq 160 \mathrm{~mm} \mathrm{Hg})$ and lower SBP $(<130 \mathrm{~mm} \mathrm{Hg})$, as well as higher DBP $(\geq 80 \mathrm{~mm} \mathrm{Hg})$ and lower DBP $(<70 \mathrm{~mm} \mathrm{Hg})[12]$. This was somewhat consistent for the very elderly, aged 75 and older, where SBP was significantly associated with MMSE in a U-shaped fashion and there was also a linear relationship with PP but no association with DBP [13]. What is distinctive in the current study is that it includes a large sample of the very elderly, and applies GAMs and the quadratic models identify cut-points that more naturally describe the shape of the relationship between blood pressure and cognitive impairment.

High PP in older adults is recognized as a marker of increased artery stiffness and widespread atherosclerosis [32], and may lead to decreased perfusion of the cerebral white matter and ultimately increase the risk for cognitive impairment as seen in some previous studies [2123]. A U-shaped association between PP and cognitive impairment found in a previous study [20], was also found in GAMs, quadratic models and univariate logistic models, but not confirmed when logistic models were further performed in our study.

It has been speculated that low or high blood pressure may be an early manifestation of the same pathological changes that lead to cognitive impairment, that is, damage to the brain regions that regulate blood pressure [33]. The association between blood pressure and cognitive impairment is problematic, and may be an effect of intermediate confounding factors affecting both blood pressure and cognitive performance such as weight loss and dietary change $[\underline{34}, \underline{35}]$, or an effect of shared risk factors such as psychological stress and oxidative stress [ $\underline{36}-\underline{38}$ ]. Another hypothesis is the healthy survivor bias. Probably, some people whose blood pressure was too low or too high had died before aged 65 years due to a window of susceptibility to related diseases (such as hemorrhagic or ischemic stroke) in middle age, thus, the elderly in our study were a selected middle blood pressure population whose health status was better, including a better cognitive performance.

Notably, the explanations and mechanisms of how both higher blood pressure and lower blood pressure impairs cognitive function are likely to be different.

\section{High blood pressure was associated with cognitive impairment}

The association between hypertension and cognitive performance may be atherosclerosis, cerebral hypoperfusion and white matter lesions [39-43]. Hypertension is a powerful risk factor for atherosclerosis throughout the arterial tree [40], which leads to redistribution of blood flow and further decrease in cerebral perfusion [41]. This phenomenon has been shown to be exceedingly important for cerebral metabolism and cognition [42]. Additionally, studies have indicated relationships between abnormalities of the white matter and hypertension [43], and such white matter lesions are frequently associated with cognitive impairment [42].

Abnormalities of the renin angiotensin system have also been involved in the pathogenesis of both hypertension and cognitive impairment. Also, in recent years antihypertensive drugs have been used in the treatment and prevention of Alzheimer's disease via the reninangiotensin system [44]. It is also hypothesized that hypoperfusion-hypoxia might accelerate cognitive impairment through inducing expression of vasoactive mediators such as nitric oxide, hypoxia-inducible-factor- $1 \alpha$, and vascular endothelial growth factor $\underline{45}$.

\section{Low blood pressure was associated with cognitive impairment}

For obvious reasons, low blood pressure can cause cerebral hypoperfusion [46]. It had also been argued that decline in blood pressure may reduce the velocity of cerebral blood flow, 
which can further cause damage to neural tissue and add to the pathological mechanisms of cognitive impairment. Reduced cerebral blood flow has been found both in individuals with dementia and in those showing early signs of dementia [46, 47]. Furthermore, it has been proposed that severe atherosclerosis resulting from long-standing low blood pressure in midlife and later-life may lead to cerebral hypoperfusion, which may be two biological pathways [48]. The review by Duschek and colleagues suggested the mechanisms of hypotension for reducing cognitive performance include diminished cortical activity, deficient regulation of cerebral blood flow in persons with low blood pressure, and insufficient adjustment of blood flow to cognitive requirements [49]. However, most studies focus on possible mechanisms of cognitive impairment caused by low blood pressure; we haven't found related researches on the mechanism of lower blood pressure within the normotensive range.

\section{Limitations}

Our study has several limitations. First, it is not possible to assess the direction of the association since both exposure and outcome are assessed simultaneously. Second, the blood pressure measurement took place at a single visit and missing data on use of antihypertensive medications may diminish the data accuracy. Third, our findings may not be applicable to younger populations because CLHLS over-sampled the oldest-old and dynamics of blood pressure changes with age. Finally, weight loss and dietary change, psychological stress and an oxidative stress may be confounding factors, but they were not measured and controlled in this study.

\section{Conclusions}

Our results suggest a U-shaped association between blood pressure (including SBP, DBP, MAP) and cognitive functions, that is, both higher and lower levels of blood pressure were associated with higher risk of cognitive impairment, which may have several implications for clinical practice and cognitive impairment prevention. First, both lower and higher blood pressure may be used to identify the high-risk population for cognitive impairment.

Furthermore, it may point to the need to individualize blood pressure management for cognitive impairment prevention, that is, we should not only address blood pressure-lowering therapy in elderly with hypertension, but may also focus on raising blood pressure in elderly with relatively lower blood pressure. Future prospective longitudinal studies with blood pressure monitoring and extensive cognitive testing are needed to confirm the association we found in this study.

\section{Acknowledgements}

The authors thank the staff of local county Centers for Disease control and Prevention in the study sites for collecting study data, and thank study participants.

\section{Funding}

This work was supported by the National Natural Science Foundation of China (grants 71110107025,81273160 and 81573247) the National Institute of Health/National Institute of Aging (grant number RO1AG023627), the Claude D. Pepper Older Americans Independence Centers (grant number 5P30 AG028716 from NIA), and Singapore Translational Research Investigator Award grant from the National Medical Research Council.

\section{Conflicts of Interest: None.}


Ethical Approval: Informed consent was obtained from all participants and/or their relatives, and the study was approved by the Ethics Committee of Peking University.

\section{References}

1. Abete P, Della-Morte D, Gargiulo G, et al. Cognitive impairment and cardiovascular diseases in the elderly. A heart-brain continuum hypothesis. Ageing Res Rev. 2014;18:4152.

2. Gottesman RF, Schneider AL, Albert M, et al. Midlife hypertension and 20-year cognitive change: the atherosclerosis risk in communities neurocognitive study. JAMA Neurol. 2014;71:1218-1227.

3. Skoog I, Lernfelt B, Landahl S, et al. 15-year longitudinal study of blood pressure and dementia. Lancet. 1996;347:1141-1145.

4. Paran E, Anson O, Reuveni $\mathrm{H}$. Blood pressure and cognitive functioning among independent elderly. Am J Hypertens. 2003;16:818-826.

5. Molander L, Gustafson Y, Lövheim H. Longitudinal associations between blood pressure and dementia in the very old. Dement Geriatr Cogn Disord. 2010;30:269-276.

6. Gelber RP, Ross GW, Petrovitch H, Masaki KH, Launer LJ, White LR. Antihypertensive medication use and risk of cognitive impairment: the Honolulu-Asia Aging

Study. Neurology. 2013;81:888-895.

7. Anderson C, Teo K, Gao P, et al. Renin-angiotensin system blockade and cognitive function in patients at high risk of cardiovascular disease: Analysis of data from the ONTARGET and TRANSCEND studies. Lancet Neurol. 2011;10:43-53.

8. Morris MC, Scherr PA, Hebert LE, Glynn RJ, Bennett DA, Evans DA. Association of incident Alzheimer disease and blood pressure measured from 13 years before to 2 years after diagnosis in a large community study. JAMA Neurol. 2001;58:1640-1646.

9. Posner HB, Tang MX, Luchsinger J, Lantigua R, Stern Y, Mayeux R. The relationship of hypertension in the elderly to $\mathrm{AD}$, vascular dementia, and cognitive function. Neurology. 2002;58:1175-1181.

10. Morris MC, Scherr PA, Hebert LE, et al. Association between Blood Pressure and Cognitive Function in a Biracial Community Population of Older Persons. Neuroepidemiology. 2002;21:123-130.

11. Waldstein SR, Giggey PP, Thayer JF, Zonderman AB. Nonlinear relations of blood pressure to cognitive function: the Baltimore Longitudinal Study of

Aging. Hypertension. 2005;45:374-379.

12. Glynn RJ, Beckett LA, Hebert LE, Morris MC, Scherr PA, Evans DA. Current and remote blood pressure and cognitive decline. JAMA. 1999;281:438-445.

13. Molander L, Gustafson Y, Lövheim H. Low blood pressure is associated with cognitive impairment in very old people. Dement Geriatr Cogn Disord. 2010;29:335-341. 
14. Thorvaldsson V, Skoog I, Hofer SM, et al. Nonlinear Blood Pressure Effects on Cognition in Old Age: Separating Between-Person and Within-Person Associations. Psychol Aging. 2011;27:375-383.

15. Waldstein SR, Katzel LI. Interactive relations of central versus total obesity and blood pressure to cognitive function. Int J Obes (Lond) 2006;30:201-207.

16. Gunstad J, Keary TA, Spitznagel MB, et al. Blood pressure and cognitive function in older adults with cardiovascular disease. Int J Neurosci. 2009;119:2228-2242.

17. Bohannon AD, Fillenbaum GG, Pieper CF, Hanlon JT, Blazer DG. Relationship of race/ethnicity and blood pressure to change in cognitive function. J Am Geriatr Soc. 2002;50:424-429.

18. Franklin SS, Khan SA, Wong ND, Larson MG, Levy D. Is pulse pressure useful in predicting risk for coronary heart Disease? The Framingham heart study. Circulation. 1999;100:354-360.

19. Franklin SS, Gustin W, Wong ND, et al. Hemodynamic patterns of age-related changes in blood pressure. The Framingham Heart Study. Circulation. 1997;96:308-315.

20. Qiu C, Winblad B, Viitanen M, Fratiglioni L. Pulse pressure and risk of Alzheimer disease in persons aged 75 years and older: a community-based, longitudinal study. Stroke. 2003;34:594-599.

21. Obisesan TO, Obisesan OA, Martins S, et al. High Blood Pressure, Hypertension, and High Pulse Pressure Are Associated with Poorer Cognitive Function in Persons Aged 60 and Older: The Third National Health and Nutrition Examination Survey. J Am Geriatr Soc. 2008;56:501-509.

22. Waldstein SR, Rice SC, Thayer JF, Najjar SS, Scuteri A, Zonderman AB. Pulse pressure and pulse wave velocity are related to cognitive decline in the Baltimore Longitudinal Study of Aging. Hypertension. 2008;51:99-104

23. Leea AY, Jeonga SH, Choib BH, Sohna EH, Chuic H. Pulse pressure correlates with leukoaraiosis in Alzheimer disease. Arch Gerontol. Geriatr. 2006;42:157-166.

24. Freitag MH, Peila R, Masaki K, et al. Midlife pulse pressure and incidence of dementia: the Honolulu-Asia Aging Study. Stroke. 2006;37:33-37.

25. Sesso HD, Stampfer MJ, Rosner B, et al. Systolic and diastolic blood pressure, pulse pressure, and mean arterial pressure as predictors of cardiovascular disease risk in Men. Hypertension. 2000;36:801-807.

26. Avanzini F, Alli C, Boccanelli A, et al. High pulse pressure and low mean arterial pressure: two predictors of death after a myocardial infarction. J Hypertens. 2006;24:23772385 .

27. Zeng Y. Towards deeper research and better policy for healthy aging --using the unique data of Chinese longitudinal healthy longevity survey. China Economic J. 2012;5:131-149.

28. Yin Z, Shi X, Kraus VB, et al. Gender-dependent association of body mass index and waist circumference with disability in the chinese oldest old. Obesity. 2014;22:1918-1925. 
29. Katzman R, Zhang M, Ouang-Ya-Qu, et al. A Chinese version of the mini-mental state examination; Impact of illiteracy in a Shanghai dementia survey. J Clin.

Epidemiol. 1988;41:971-978.

30. Moore DJ, Palmer BW, Jeste DV. Use of the Mini-Mental State Exam in Middle-Aged and Older Outpatients With Schizophrenia : Cognitive Impairment and Its Associations. Am J Geriatr Psychiatry. 2004;12:412-419.

31. Tombaugh TN, Mcintyre NJ. The mini-mental state examination: a comprehensive review. J Am Geriatr Soc. 1992;40:922-935

32. Mitchell GF, Vasan RS, Keyes MJ, et al. Pulse pressure and risk of new-onset atrial fibrillation. JAMA. 2007;297:709-715.

33. Burke WJ, Coronado PG, Schmitt CA, Gillespie KM, Chung HD. Blood pressure regulation in alzheimer's disease. J Auton Nerv Syst. 1994;48:65-71.

34. Gale CR, Martyn CN, Cooper C. Cognitive impairment and mortality in a cohort of elderly people. BMJ. 1996;312:608-611.

35. White H, Pieper C, Schmader K, Fillenbaum G. Weight Change in Alzheimer's Disease. J Am Geriatr Soc. 1996;44:265-272.

36. Carroll D, Phillips AC, Der G, Hunt K, Benzeval M. Blood pressure reactions to acute mental stress and future blood pressure status: data from the 12-year follow-up of the West of Scotland Study. Psychosom Med. 2011;73:737-742.

37. Marin MF, Lord C, Andrews J, et al. Chronic stress, cognitive functioning and mental health. Neurobiol Learn Mem. 2011;96:583-595.

38. Kim HA, Miller AA, Drummond GR, et al. Vascular cognitive impairment and Alzheimer's disease: role of cerebral hypoperfusion and oxidative stress. Naunyn Schmiedebergs Arch Pharmacol. 2012;385:953-959.

39. Bos D, Vernooij MW, de Bruijn RF, et al. Atherosclerotic calcification is related to a higher risk of dementia and cognitive decline. Alzheimers Dement. 2014;11:639-647. e631.

40. Arnett DK, Boland LL, Evans GW, et al. Hypertension and arterial stiffness: the atherosclerosis risk in communities study. Am J Hypertens. 2000;13:317-323.

41. de la Torre JC, Stefano GB. Evidence that Alzheimer's disease is a microvascular disorder: the role of constitutive nitric oxide. Brain Res Brain Res Rev. 2000;34:119-136.

42. Gurol ME. Cerebral hypoperfusion and white matter disease in healthy elderly and patients with Alzheimer's disease. Eur J Neurol. 2013;20:214-215.

43. Verhaaren BF, Vernooij MW, Renske B, et al. High Blood Pressure and Cerebral White Matter Lesion Progression in the General Population. Hypertension. 2013;61:1354-1359.

44. Kehoe PG, Wilcock GK. Is inhibition of the renin-angiotensin system a new treatment option for Alzheimer's disease? Lancet Neurol. 2007;6:373-378.

45. Takeda S, Sato N, Ogihara T, Morishita R. The renin-angiotensin system, hypertension and cognitive dysfunction in Alzheimer's disease: new therapeutic potential. Front Biosci. 2008;13:2253-2265. 
46. Kalback W, Esh C, Castaño EM, et al. Atherosclerosis, vascular amyloidosis and brain hypoperfusion in the pathogenesis of sporadic Alzheimer's disease. Neurol

Res. 2004;26(5):525-539

47. Launer LJ, Masaki K, Petrovitch H, Foley D, Havlik RJ. The association between midlife blood pressure levels and late-life cognitive function. The Honolulu-Asia Aging Study. JAMA. 1995;274:1846-1851.

48. Qiu C, Winblad B, Fratiglioni L. The age-dependent relation of blood pressure to cognitive function and dementia. Lancet Neurol. 2005;4:487-499.

49. Duschek S, Schandry R. Reduced brain perfusion and cognitive performance due to constitutional hypotension. Clin Auton Res. 2007;17:69-76. 
Table 1

Characteristics of 7,144 Chinese elderly by cognitive status, 2011

\begin{tabular}{|c|c|c|c|c|}
\hline Factors $^{*}$ & $\begin{array}{l}\text { Normal } \\
\text { cognition } \\
n=4393\end{array}$ & $\begin{array}{l}\text { Cognitive } \\
\text { impairment } \\
\mathrm{n}=2751\end{array}$ & $\begin{array}{l}\text { Total } \\
\mathrm{n}=7144\end{array}$ & P-value \\
\hline MMSE & $26.8(2.3)$ & $9.2(6.7)$ & 21.1(10.9) & $<0.0001$ \\
\hline Age (year) & $83.3(9.7)$ & $93.2(9.7)$ & $87.1(10.9)$ & $<0.0001$ \\
\hline \multicolumn{5}{|l|}{ Gender } \\
\hline Male & 2335(46.9) & $900(32.7)$ & $3235(45.3)$ & $<0.0001$ \\
\hline Female & $2058(53.1)$ & 1851(67.3) & $3909(54.7)$ & \\
\hline \multicolumn{5}{|l|}{ Residence } \\
\hline Urban & $2226(59.8)$ & $1464(53.2)$ & $4430(57.2)$ & $<0.0001$ \\
\hline Rural & 1787(40.2) & $1287(46.8)$ & $3054(42.8)$ & \\
\hline Education (year) & $3.2(3.0)$ & $1.1(2.5)$ & $2.4(3.6)$ & $<0.0001$ \\
\hline Physically active & $2100(48.0)$ & $671(24.5)$ & $2771(39.0)$ & $<0.0001$ \\
\hline Good visual function & $3749(85.3)$ & $1577(57.3)$ & $5326(74.6)$ & $<0.0001$ \\
\hline Central obesity & $2198(50.0)$ & $1102(40.1)$ & $3300(46.2)$ & $<0.0001$ \\
\hline Diabetes mellitus & $248(5.9)$ & $92(3.7)$ & $340(5.1)$ & $<0.0001$ \\
\hline Cardiovascular diseases & $653(15.5)$ & $324(12.8)$ & $977(14.5)$ & 0.0024 \\
\hline $\begin{array}{l}\text { Stroke and cerebrovascular } \\
\text { diseases }\end{array}$ & $332(7.9)$ & $265(10.4)$ & $597(8.8)$ & 0.0004 \\
\hline $\begin{array}{l}\text { Systolic blood pressure } \\
(\mathrm{mmHg})\end{array}$ & $135.7(19.4)$ & $135.2(22.0)$ & $135.5(20.4)$ & 0.3055 \\
\hline $\begin{array}{l}\text { Diastolic blood pressure } \\
(\mathrm{mmHg})\end{array}$ & $80.3(11.2)$ & $79.8(12.9)$ & $80.1(11.9)$ & 0.1302 \\
\hline Pulse pressure $(\mathrm{mmHg})$ & $55.4(15.9)$ & $55.4(17.5)$ & $55.4(16.6)$ & 0.8600 \\
\hline $\begin{array}{l}\text { Mean arterial } \\
\text { pressure }(\mathrm{mmHg})\end{array}$ & $98.8(12.3)$ & $98.3(14.3)$ & $98.6(13.1)$ & 0.1494 \\
\hline Hypertension & $1859(42.3)$ & $1162(42.2)$ & $3021(42.3)$ & 0.9481 \\
\hline
\end{tabular}

"Dichotomous variables listed as n (\%), including gender, current smoking practice, alcohol drinking habits, central obesity, diabetes, cardiovascular diseases, stroke and cerebrovascular diseases, hypertension, were compared between two cognitive status groups using Chi-square tests; Continuous variables listed as mean (standard deviation), including age, education, SBP, DBP, PP, MAP, were comparted between two groups using Student's T tests or Cochran-Cox tests. MMSE, Mini Mental State Examination. 
Table 2

Logistic odds ratio (95\% Confidence Intervals) for cognitive impairment and continuous measures of blood pressure among Chinese elderly.

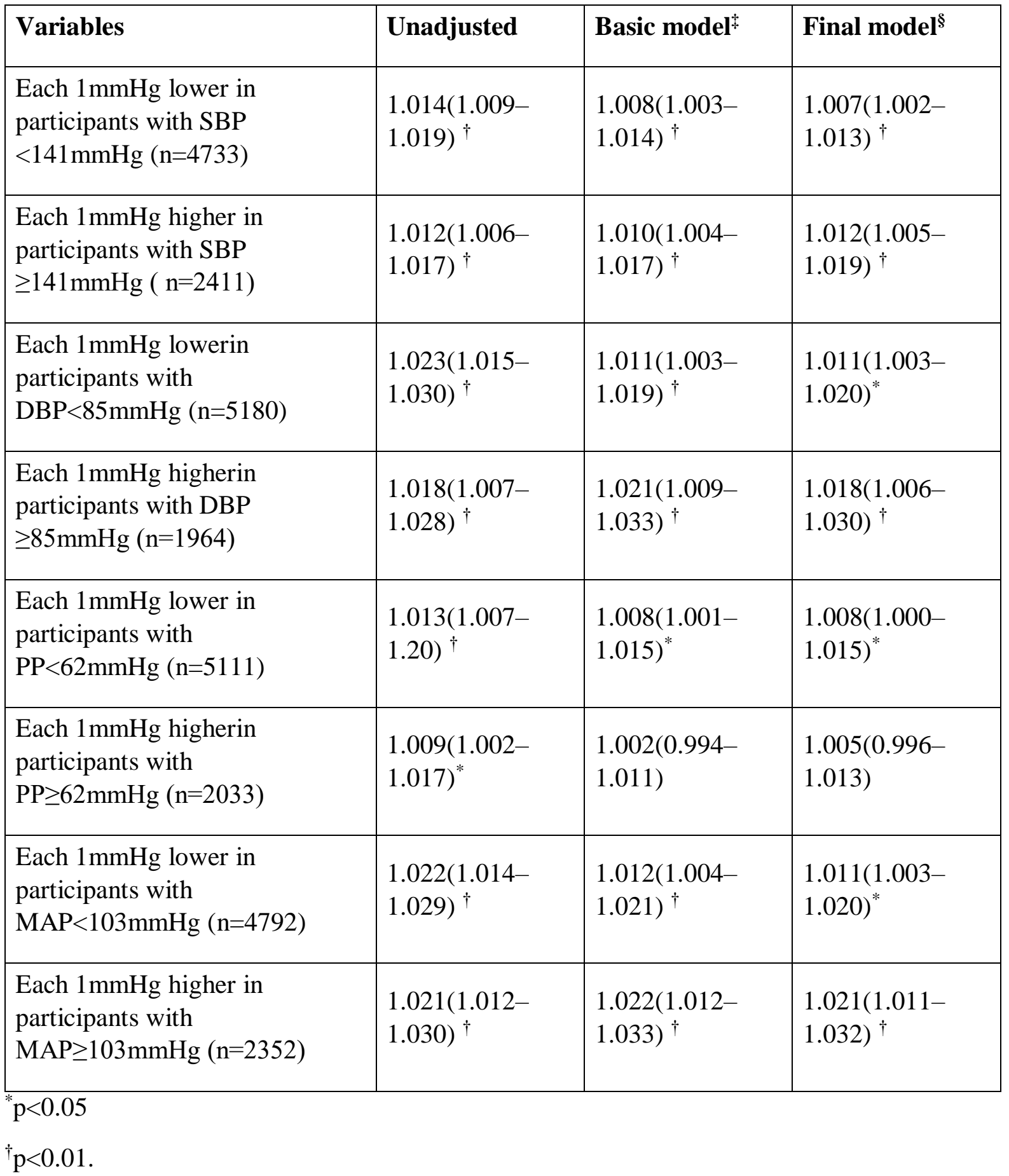

*Adjusted for demographic variables including age (year), gender, education level (year), residence in basic model,

${ }^{\S}$ Further adjusted physical activity, vision, waist circumference, type 2 diabetes mellitus, cardiovascular diseases and stroke and cerebrovascular diseases in final model. 
DBP diastolic blood pressure; MAP mean arterial pressure, PP pulse pressure, SBP systolic blood pressure. 
Table 3

Logistic odds ratio (95\% confidence intervals) for cognitive impairment and blood pressure categories among Chinese elderly. ${ }^{\dagger}$

\begin{tabular}{|c|c|c|c|}
\hline Variables & Unadjusted & Basic model ${ }^{\sharp}$ & Final model§ \\
\hline \multicolumn{4}{|l|}{ SBP } \\
\hline Low $(\mathrm{SBP}<127 \mathrm{mmHg}, \mathrm{n}=2324)$ & $\begin{array}{l}1.25(1.12- \\
1.41)^{\dagger}\end{array}$ & $\begin{array}{l}1.15(1.00- \\
1.32)^{*}\end{array}$ & $\begin{array}{l}1.18(1.02- \\
1.35)^{*}\end{array}$ \\
\hline $\begin{array}{l}\text { Medium-low }(127 \leq \mathrm{SBP}<141 \mathrm{mmHg} \text {, } \\
\mathrm{n}=2409)\end{array}$ & 1.00 reference & 1.00 reference & 1.00 reference \\
\hline $\begin{array}{l}\text { Medium-high }(141 \leq \mathrm{SBP}<155 \mathrm{mmHg} \text {, } \\
\mathrm{n}=1284)\end{array}$ & 1.00 reference & 1.00 reference & 1.00 reference \\
\hline $\operatorname{High}(\mathrm{SBP} \geq 155 \mathrm{mmHg}, \mathrm{n}=1127)$ & $\begin{array}{l}1.21(1.02- \\
1.42)^{*}\end{array}$ & $\begin{array}{l}1.18(0.99- \\
1.42)\end{array}$ & $\begin{array}{l}1.23(1.02- \\
1.49)^{*}\end{array}$ \\
\hline \multicolumn{4}{|l|}{ DBP } \\
\hline Low $(\mathrm{DBP}<77 \mathrm{mmHg}, \mathrm{n}=2536)$ & $\begin{array}{l}1.33(1.19- \\
1.49)^{\dagger}\end{array}$ & $\begin{array}{l}1.20(1.05- \\
1.36)^{\dagger}\end{array}$ & $\begin{array}{l}1.20(1.05- \\
1.37)^{\dagger}\end{array}$ \\
\hline $\begin{array}{l}\text { Medium-low }(77 \leq \mathrm{DBP}<85 \mathrm{mmHg} \text {, } \\
\mathrm{n}=2644)\end{array}$ & 1.00 reference & 1.00 reference & 1.00 reference \\
\hline $\begin{array}{l}\text { Medium-High }(85 \leq \mathrm{DBP}<90 \mathrm{mmHg} \text {, } \\
\mathrm{n}=989)\end{array}$ & 1.00 reference & 1.00 reference & 1.00 reference \\
\hline $\operatorname{High}(\mathrm{DBP} \geq 90 \mathrm{mmHg}, \mathrm{n}=975)$ & $\begin{array}{l}1.26(1.07- \\
1.50)^{\dagger}\end{array}$ & $\begin{array}{l}1.26(1.04- \\
1.52)^{*}\end{array}$ & $\begin{array}{l}1.24(1.02- \\
1.52)^{*}\end{array}$ \\
\hline \multicolumn{4}{|l|}{ PP } \\
\hline Low $(\mathrm{PP}<49 \mathrm{mmHg}, \mathrm{n}=2523)$ & $\begin{array}{l}1.18(1.05- \\
1.32)^{\dagger}\end{array}$ & $\begin{array}{l}1.08(0.95- \\
1.23)\end{array}$ & $\begin{array}{l}1.09(0.95- \\
1.25)\end{array}$ \\
\hline $\begin{array}{l}\text { Medium-low }(49 \leq \mathrm{PP}<62 \mathrm{mmHg}, \\
\mathrm{n}=2588)\end{array}$ & 1.00 reference & 1.00 reference & 1.00 reference \\
\hline $\begin{array}{l}\text { Medium-high }(62 \leq \mathrm{PP}<73 \mathrm{mmHg} \text {, } \\
\mathrm{n}=1035)\end{array}$ & 1.00 reference & 1.00 reference & 1.00 reference \\
\hline High $(P P \geq 73 m m H g, n=998)$ & $\begin{array}{l}1.04(0.87- \\
1.24)\end{array}$ & $\begin{array}{l}0.90(0.74- \\
1.10)\end{array}$ & $\begin{array}{l}1.04(0.84- \\
1.28)\end{array}$ \\
\hline
\end{tabular}




\begin{tabular}{|c|c|c|c|}
\hline Variables & Unadjusted & Basic model $^{\ddagger}$ & Final model§ \\
\hline \multicolumn{4}{|l|}{ MAP } \\
\hline Low(MAP<93mmHg, n=2560) & $\begin{array}{l}1.31(1.17- \\
1.48)^{\dagger}\end{array}$ & $\begin{array}{l}1.15(1.00- \\
1.33)\end{array}$ & $\begin{array}{l}1.15(1.00- \\
1.34)^{*}\end{array}$ \\
\hline $\begin{array}{l}\text { Medium-low }(93 \leq \mathrm{MAP}<103 \mathrm{mmHg} \text {, } \\
\mathrm{n}=2232)\end{array}$ & 1.00 reference & 1.00 reference & 1.00 reference \\
\hline $\begin{array}{l}\text { Medium-high }(103 \leq \mathrm{MAP}<110 \mathrm{mmHg} \text {, } \\
\mathrm{n}=1 \text { 186) }\end{array}$ & 1.00 reference & 1.00 reference & 1.00 reference \\
\hline High $(\mathrm{MAP} \geq 110 \mathrm{mmHg}, \mathrm{n}=1166)$ & $\begin{array}{l}1.21(0.99- \\
1.33)\end{array}$ & $\begin{array}{l}1.20(0.99- \\
1.44)\end{array}$ & $\begin{array}{l}1.22(1.01- \\
1.48)^{*}\end{array}$ \\
\hline
\end{tabular}

${ }^{*} \mathrm{p}<0.05$

${ }^{\dagger} \mathrm{p}<0.01$.

Adjusted for demographic variables including age (year), gender, education level (year), residence in basic model, §Further adjusted physical activity, vision, waist circumference, type 2 diabetes mellitus, cardiovascular diseases and stroke and cerebrovascular diseases in final model.

DBP diastolic blood pressure; MAP mean arterial pressure, PP pulse pressure, SBP systolic blood pressure. 
Figure 1

\section{Smoothing component for cognitive impairment}

Legends: Associations of SBP, DBP, PP and MAP with cognitive impairment for the elderly, Chinese Longitudinal Healthy Longevity Survey, China, 2011. The lines depict the estimated function of SBP, DBP, PP and MAP respectively, for risk of cognitive impairment among the elderly, and the shaded area indicates the $95 \%$ confidence intervals.

SBP, Systolic blood pressure; DBP, diastolic blood pressures; PP, pulse pressure; MAP, mean arterial pressure.

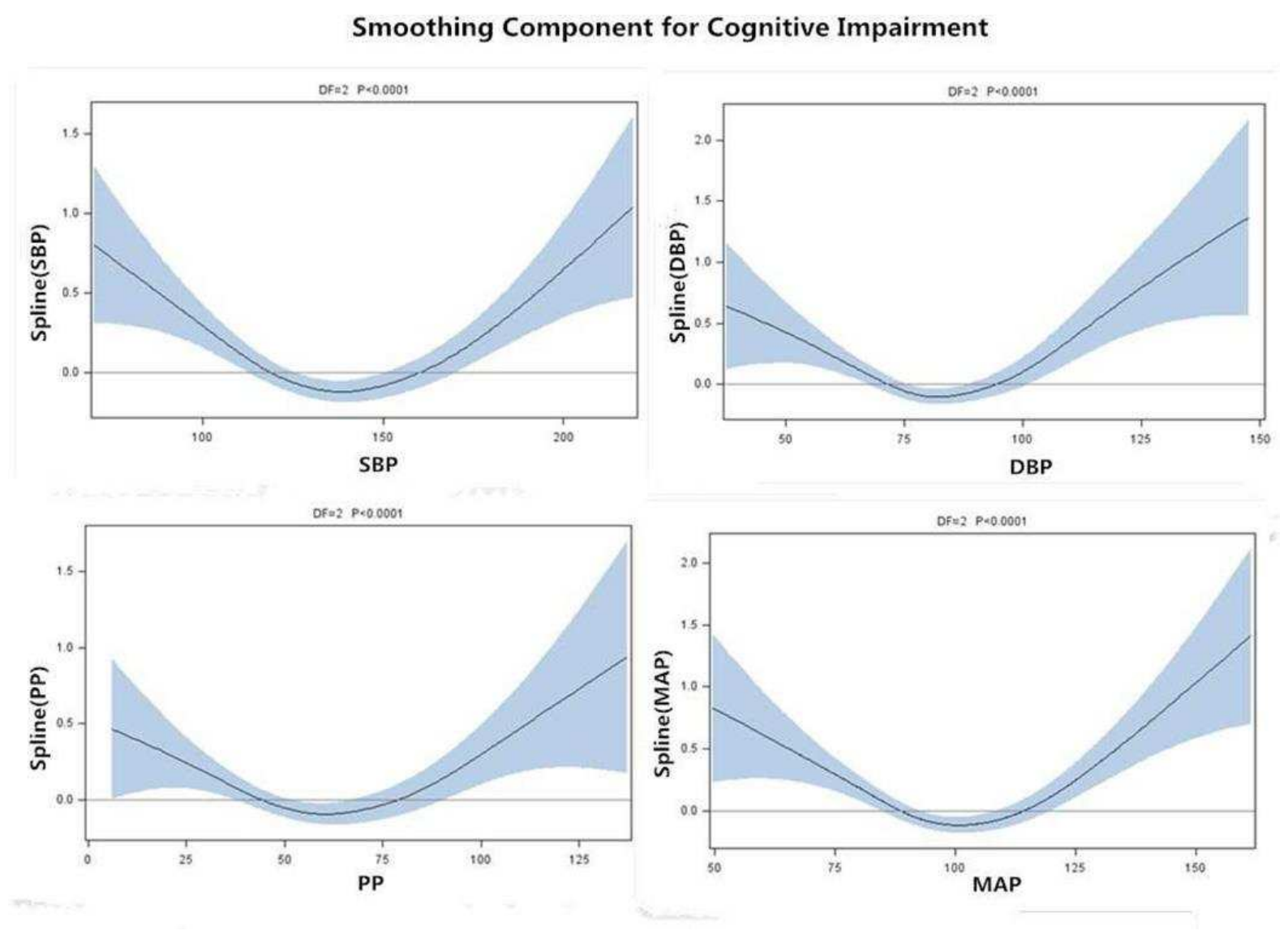

